\title{
Un marco analítico para la evaluación de la calidad de la gobernanza de los sistemas educativos
}

\author{
Francisco López Rupérez 1 (D) @ \\ Isabel García García ${ }^{1}$ (D) @ \\ Eva Expósito-Casas ${ }^{2}$ (1) @ \\ ${ }^{1}$ Universidad Camilo José Cela (UCJC), España; ${ }^{2}$ Universidad Nacional de Educación a Distancia (UNED), España
}

Resumen. En el momento presente los sectores más informados de la sociedad dirigen su mirada hacia los sistemas de educación y formación con la esperanza de que éstos contribuyan a aportar una respuesta adaptativa a los desafíos del nuevo contexto histórico. A causa de tales desafíos personales, sociales y económicos, nunca como ahora la exigencia de acierto, en la definición y la implementación de las políticas educativas, había sido tan perentoria. El presente trabajo plantea la elaboración de un marco analítico para la evaluación de la calidad de la gobernanza educativa, mediante el desarrollo de un sistema de indicadores que reposa en un marco conceptual robusto, previamente definido. Este instrumento de diagnóstico ha sido refinado y validado mediante un procedimiento Delphi de tres rondas sucesivas y se ha estructurado finalmente en 6 dimensiones, 20 subdimensiones y 88 ítems. Un estudio exploratorio de aplicación de la herramienta al caso español ha permitido poner a prueba su fiabilidad. A partir de los resultados empíricos obtenidos del estudio Delphi, se analizan y discuten los criterios de calidad de la gobernanza educativa que han resultado ser más relevantes, y se extraen algunas conclusiones para la mejora de la educación.

Palabras clave: Gobernanza educativa; método Delphi; indicadores de calidad; evaluación.

Um marco analítico para a avaliação da qualidade da governança dos sistemas educacionais Resumo. No momento presente, os setores mais informados da sociedade dirigem seu olhar para os sistemas de educação e de formação, com a esperança de que esses contribuam para dar uma resposta adaptável aos desafios do novo contexto histórico. Por causa desses desafios pessoais, sociais e econômicos, a exigência do acerto na definição e na implementação das políticas educacionais nunca tinha sido tão terminante como agora. O presente trabalho propõe a elaboração de um marco analítico para a avaliação da qualidade da governança da educação, através do desenvolvimento de um sistema de indicadores apoiado num mapa conceitual bem estruturado, previamente definido. Este instrumento de diagnóstico foi refinado e legitimado por meio do método Delphi de três rodadas sucessivas e finalmente foi estruturado em 6 dimensões, 20 subdimensões e 88 itens. Um estudo exploratório da ferramenta aplicado ao caso espanhol permitiu pôr à prova sua fiabilidade. A partir dos resultados empíricos obtidos do estudo Delphi, analisam-se e discutem-se os critérios de qualidade da governança educacional que foram mais relevantes, chegando a algumas conclusões para a melhoria da educação.

Palavras-chave: Governança educacional; método Delphi; indicadores de qualidade; avaliação.

An analytical framework for the evaluation of the quality of governance of education systems Abstract. At the present time, the most well-informed sectors of society are turning their attention to education and training systems in the hope that these will contribute towards providing an adaptive response to the challenges of this new social and economic context. The need for wise choices, in terms of defining and implementing education policies, has never been so pressing. This research poses the production of an analytic framework to evaluate quality of educational governance at system level. For this, the development of a system of subjective indicators, resting on a robust previously defined conceptual framework, has been made. This system has been refined and validated by means of a three successive rounds Delphi procedure and has finally been structured into 6 dimensions, 20 subdimensions and 88 items. An exploratory study for applying the tool to the Spanish case enabled its reliability to be tested. On the basis, of the empirical results obtained, the quality criteria for educational governance that have turned out to be the most important are analysed and discussed, and conclusions are drawn on how education can be improved.

Keywords: Educational governance; Delphi method; quality indicators; evaluation. 


\section{Introducción}

En el momento actual, una buena parte de los países del mundo está asistiendo y protagonizando una revolución tecnológica, social, económica y cultural sin precedentes en tan sólo una generación: una auténtica “mutación de civilización” (López Rupérez, 2001). En esta circunstancia, los sectores más informados de la sociedad dirigen su mirada hacia los sistemas de educación y formación con la esperanza de que contribuyan a aportar una respuesta adaptativa a los desafíos de este nuevo contexto histórico. A causa de tales desafíos, nunca como ahora la exigencia de acierto, en el ámbito de la definición y de la implementación de las políticas educativas, había sido tan perentoria, ni su reconocimiento tan extendido.

Este fenómeno no es exclusivo, en modo alguno, de los países más desarrollados, sino que se extiende a otros países de menor nivel de desarrollo cuyos dirigentes, con el apoyo de organismos multilaterales como la UNESCO, la OCDE o la OEI, han visto en la mejora de sus sistemas de educación y formación una palanca formidable de transformación social y económica. Así, en la edición de 2015 de PISA, diez países de Iberoamérica -incluyendo México, Chile y Colombia, miembros de la OCDE en la actualidad-participaron en esa evaluación internacional a gran escala. Y es que una aproximación transversal a las políticas educativas va ganado terreno en el mundo; o, dicho de otro modo, y como la propia OCDE ha postulado, una "globalización de las políticas" (Schleicher, 2018) avanza progresivamente.

Desde una aproximación multinivel, cabe subrayar la existencia de una abundante evidencia empírica, procedente de síntesis meta-analíticas (Hattie, 2003, 2009, 2017), sobre el impacto de la calidad del profesor en los resultados de los alumnos en el nivel micro propio del aula (nivel 1). Así mismo, se dispone de un cuerpo de evidencia suficientemente robusto que muestra el notable impacto del liderazgo de la dirección en el nivel meso o del centro educativo (nivel 2); impacto que se sitúa, en cuanto a magnitud, inmediatamente después del correspondiente al profesor (Leithwood et al., 2004.; Leithwood et al., 2012; Hallinger, 2014; Hanushek et al., 2016; Pont Ferrer 2017; López Rupérez et al., 2020). Sin embargo, no existe una estimación de la magnitud del impacto de la calidad de la gobernanza del sistema educativo en su conjunto (nivel 3 ) sobre los outputs de dicho sistema. Ello es así, entre otras razones, porque carecemos, por el momento, de instrumentos válidos y fiables para evaluar la calidad del liderazgo en ese nivel 3, o nivel macro. Sin embargo, incluso en los países más descentralizados en materia educativa, existe un cierto protagonismo del nivel 3 que, por su elevada posición jerárquica, propaga su influencia, como en cascada, en los niveles inferiores y puede generar avances sustantivos en la educación, en la economía y en la sociedad. Resulta a este respecto muy descriptivo el 
caso de Finlandia en donde opera la máxima "dirección central, decisiones locales" (Lamb et al., 2017). Trasponiendo el razonamiento de Hanushek et al. (2016) del nivel 2 hacia el nivel 3, cabe incluso conjeturar si la mejora de la calidad de la gobernanza ${ }^{1}$ de los sistemas educativos, no podría acaso generar unos beneficios superiores a los que se obtienen por la mejora de la calidad del profesor o del director, probablemente por razones no de intensidad, pero sí de extensión; pura y simplemente porque afectaría a muchos más alumnos.

La investigación que aquí se presenta ha emprendido la tarea de elaborar un marco analítico para la evaluación de la calidad de la gobernanza de los sistemas educativos que reposa en un sistema de indicadores subjetivos apoyado, a su vez, en un marco conceptual robusto, previamente definido. Este instrumento, que ha sido refinado y validado mediante un procedimiento Delphi de tres rondas sucesivas, se ha estructurado finalmente en 6 dimensiones, 20 subdimensiones y 88 ítems. Un estudio exploratorio de aplicación de la herramienta al caso español (López Rupérez et al., 2017) ha permitido poner a prueba su fiabilidad.

En lo que sigue, se describe la metodología empleada en la elaboración de ese instrumento analítico de evaluación, se presentan los resultados empíricos obtenidos, se analizan y discuten los criterios de calidad de la gobernanza educativa que han resultado ser más relevantes, y se extraen algunas conclusiones para la mejora de la educación. La proximidad cultural del contexto español -propio de la elaboración de dicho instrumento- con respecto a la región iberoamericana, en general, e hispanoamericana, en particular, podría hacerlo útil para su aplicación en esta región o en alguno de sus países componentes.

\section{Métodos}

En lo que concierne a la metodología, es preciso distinguir dos procedimientos consecutivos, pero bien diferenciados entre sí, que han sido empleados en el presente estudio: por un lado, la elaboración de la versión inicial del cuestionario y sus bases conceptuales, y, por otro, la aplicación de un procedimiento Delphi para su refinamiento y validación.

${ }^{1}$ Se entiende aquí por gobernanza: "La capacidad de los gobiernos para diseñar, formular e implementar políticas y desempeñar sus funciones”, (World Bank, 1994, p. xiv). 


\subsection{La elaboración de la versión inicial del cuestionario y sus bases conceptuales}

La elaboración de la versión cero, o inicial, del cuestionario, se ha efectuado a partir de un conjunto de 6 categorías y 20 subcategorías de indicadores procedentes de un marco en el que se han integrado los criterios resultantes de tres análisis conceptuales diferentes: el que corresponde a la trasposición al ámbito educativo de una perspectiva "clásica" sobre la gobernanza del sector público (A) (World Bank, 1994; Hewitt, 1998; United Nations,2007; Fazekas et al., 2012; OCDE,2015); el derivado de la aplicación del paradigma de la complejidad a la gestión de los sistemas educativos (B) (López Rupérez, 1997, 2001; OECD/CERI, 2015; OECD, 2016) ("aproximación moderna"); y, finalmente, el asociado a una "gobernanza inteligente" (C) (López Rupérez et al., 2017). Todos estos análisis se muestran sintéticamente, en forma de criterios de calidad de la gobernanza educativa, en las tablas 1,2 y 3 .

Aun cuando las tres perspectivas conceptuales citadas tienen fuentes de inspiración, en lo esencial, independientes, han aparecido algunos solapamientos inevitables entre ellas. Con el fin de identificar tales coincidencias, se ha señalado en cada una de las tres tablas citadas las relaciones existentes entre cada criterio de gobernanza propio de una aproximación determinada -clásica, moderna y de "gobernanza inteligente"-y los de las otras dos aproximaciones consideradas en el trabajo.

Tabla 1. Criterios principales de gobernanza en el sector público, desde una aproximación clásica, y su transposición al sector educativo

\begin{tabular}{|c|c|c|c|c|c|c|}
\hline $\begin{array}{l}\text { A. Aproximación clásica } \\
\text { (criterios) }\end{array}$ & $\begin{array}{c}\text { World } \\
\text { Bank } \\
(1994)\end{array}$ & $\begin{array}{l}\text { Hewitt, C. } \\
\text { (1998) }\end{array}$ & $\begin{array}{l}\text { United } \\
\text { Nations } \\
(2007)\end{array}$ & $\begin{array}{l}\text { Fazekas et } \\
\text { al. (2012) }\end{array}$ & $\begin{array}{l}\text { OCDE } \\
(2015)\end{array}$ & $\begin{array}{l}\text { Relacio- } \\
\text { nado con } \\
\text { el criterio }\end{array}$ \\
\hline $\begin{array}{l}\text { A1. Construcción de } \\
\text { consensosy participación }\end{array}$ & & $x$ & $x$ & & $x$ & B4 y B5 \\
\hline $\begin{array}{l}\text { A2. Selección de respon- } \\
\text { sables }\end{array}$ & $x$ & & & & & \\
\hline $\begin{array}{l}\text { A3. Gestión eficiente de } \\
\text { recursos }\end{array}$ & $x$ & & $x$ & & & $\mathrm{~B} 6$ y $\mathrm{C} 1$ \\
\hline $\begin{array}{l}\text { A4. Desempeño de fun- } \\
\text { ciones }\end{array}$ & & & $x$ & & & B6 \\
\hline A5. Regulación & & & & & $x$ & B6 \\
\hline $\begin{array}{l}\text { A6. Definición de prio- } \\
\text { ridades }\end{array}$ & & & $x$ & $x$ & & B3 \\
\hline A7. Rendición de cuentas & & & & $x$ & & $\mathrm{~B} 2$ y $\mathrm{C} 1$ \\
\hline $\begin{array}{l}\text { A8. Capacidad de di- } \\
\text { rección }\end{array}$ & & & & $x$ & & B3 \\
\hline A9. Transparencia & & & $x$ & & & \\
\hline A10. Responsabilidad & & & $x$ & & & A7 \\
\hline
\end{tabular}

Fuente: Elaboración propia. 
Tabla 2. Criterios principales de gobernanza educativa desde una aproximación moderna que toma en consideración el paradigma de la complejidad

\begin{tabular}{cccc} 
B. & GCES & & \\
$\begin{array}{c}\text { Aproximación moderna } \\
\text { (criterios) }\end{array}$ & (OECD/ & OECD & Relacionado \\
& CERI) & $(2016)$ & con el criterio \\
& $(2015)$ & & \\
\hline
\end{tabular}

\begin{tabular}{lccc} 
B1. Construcción de capacidades & X & X & \\
\hline B2. Rendición de cuentas & X & X & A7 y C1 \\
\hline B3. Visión estratégica & X & X & A6 y A8 \\
\hline B4. Enfoque holístico y multinivel & X & X & A1 \\
\hline B5. Flexible y adaptativa & X & X & A1 y C2 \\
\hline $\begin{array}{l}\text { B6. Centrada en los procesos } \\
\text { B7. Basada en la evidencia empírica y en la } \\
\text { investigación }\end{array}$ & & X & A3, A4, A5, C3, C4 y C5 \\
\hline
\end{tabular}

B8. Concede importancia al nivel nacional $\quad \mathrm{X}$

Fuente: Elaboración propia.

Tabla 3. Criterios principales de gobernanza educativa desde una concepción de "gobernanza inteligente"

\begin{tabular}{lcc}
\hline \multicolumn{1}{c}{$\begin{array}{c}\text { C. Gobernanza inteligente } \\
\text { (criterios) }\end{array}$} & $\begin{array}{c}\text { López Rupérez } \\
\text { et al. (2017) }\end{array}$ & $\begin{array}{c}\text { Relacionado } \\
\text { con el criterio }\end{array}$ \\
\hline $\begin{array}{l}\text { C1. Importancia del feedback y de la evaluación } \\
\text { de las políticas }\end{array}$ & X & A3, A7, B2 y B7 \\
\hline C2. Respuestas adaptativas & X & B5 \\
\hline $\begin{array}{l}\text { C3. Preocupada por la implementación } \\
\begin{array}{l}\text { C4. Con implicación personal de los responsables } \\
\text { de alto nivel }\end{array}\end{array}$ & X & B6 \\
\hline $\begin{array}{l}\text { C5. Con involucración de los actores de niveles } \\
\text { inferiores, especialmente de los docentes }\end{array}$ & X & B6 \\
\hline $\begin{array}{l}\text { C6. Importancia del feedback y de la evaluación } \\
\text { de las políticas }\end{array}$ & X & A3, A7, B2 y B7 \\
\hline
\end{tabular}

Fuente: Elaboración propia.

Un análisis jerárquico sobre el conjunto de criterios enunciados en las citadas tablas y su posterior reclasificación ha permitido operativizar un marco conceptual integrado y razonablemente robusto mediante su traducción en el siguiente sistema de dimensiones y subdimensiones: 
1. Gobernanza con un enfoque holístico y multinivel (B4)

1.1 Toma en consideración la existencia de relaciones e interdependencias entre los diferentes niveles del sistema (individual, institucional y de gobierno) (B4).

1.2 Preserva la importancia del nivel estatal (B8).

1.3 Busca el consenso (A1).

1.4 Promueve la participación de los actores de niveles inferiores, principalmente del personal docente (A1 y C5).

2. Gobernanza con capacidad de dirección (A8)

2.1 Dispone de una visión estratégica (B3).

2.2 Establece con claridad las prioridades (A6).

2.3 Concede mucha importancia a la selección de los responsables de las políticas (A2).

2.4 Comporta una fuerte implicación personal de los responsables de las políticas con los procesos de mejora (C4).

3. Gobernanza centrada en los procesos (B6)

3.1 Realiza una gestión eficiente de los recursos (A3).

3.2 Ejerce correctamente sus funciones principales (A4).

3.3 Elabora una regulación apropiada y de calidad (A5).

3.4 Otorga mucha importancia a la implementación (C3).

4. Gobernanza basada en el conocimiento, la evidencia empírica y la investigación (B7)

4.1 Es flexible y adaptativa (B5 y C2).

4.2 Promueve y emplea el conocimiento y la investigación para una mejor formulación de las políticas (B7).

4.3 Otorga mucha importancia al feedback (C1).

4.4 Evalúa el impacto de las políticas (C1).

5. Gobernanza basada en la construcción de capacidades (B1)

5.1 Establece diferentes fórmulas para compartir con los actores principales el conocimiento sobre las políticas y sobre su implementación (B1).

5.2 Ayuda a los actores principales a adquirir e utilizar información relevante para el éxito en la implementación de las políticas (B1).

6. Gobernanza vinculada a la rendición de cuentas (A7 y B2)

6.1 Concede una gran importancia a la transparencia (A9).

6.2 Establece mecanismos de responsabilidad (A10). 


\subsection{El procedimiento Delphi}

La técnica Delphi (Landeta, 1999; Hasson et al., 2000; Varela Ruiz et al., 2012; Linstone et al., 2012) ha sido ampliamente utilizada en investigaciones en el área de la salud (Uphoff et al., 2012) y particularmente en la elaboración de indicadores de calidad asistencial (Boulkedid, 2011). En el ámbito de los indicadores de calidad educativa se ha empleado, asimismo, en el área de la gestión de las instituciones de educación superior y, en particular, en la definición de indicadores de gobernanza universitaria (Ngoc Quyen, 2014). Sin embargo, no hemos encontrado en la literatura especializada referencias previas relativas al empleo de dicha técnica de consulta iterativa para la identificación de indicadores de calidad de la gobernanza de los sistemas educativos.

\subsubsection{La selección del panel de expertos}

La selección del panel de expertos ha sido efectuada atendiendo a criterios tanto cuantitativos como cualitativos. En relación con los primeros, se ha tomado en consideración la minimización del "error medio grupal" (Landeta 1999). No se ha recurrido a un número excesivo de componentes del panel, con el fin de evitar que se complicara notablemente la gestión del proceso sin producirse una reducción apreciable de dicho error. De conformidad con tales criterios, se ha fijado en 21 el número de expertos del panel.

En relación con los criterios cualitativos, se ha optado por asegurar en el panel la representación de tres grupos de actores principales directamente concernidos por la calidad de la gobernanza: los responsables políticos -tanto en el nivel estatal como en el regional-, los investigadores, y los directores escolares de centros públicos de educación secundaria. Estos tres grupos de actores relevantes aportan visiones desde planos diferentes sobre la calidad de la gobernanza y sobre sus claves fundamentales. (Véase la tabla 4). De acuerdo con las recomendaciones recogidas en la literatura (Landeta 1999), la heterogeneidad del panel puede dar mejores resultados que la homogeneidad, particularmente, como es el caso, cuando existe una pluralidad de actores implicados de distinta naturaleza. Además, esta participación plural puede mejorar la credibilidad y la aceptación, por parte de sus destinatarios, del sistema de indicadores resultante del estudio Delphi (Boulkedid, 2011). 
Tabla 4. Características del panel de expertos

\begin{tabular}{|c|c|c|}
\hline Grupo & Perfil & Edad media (años) \\
\hline Ex altos cargos de Educación & $\begin{array}{l}\text { Tres exsecretarios generales de Educación } \\
\text { del Gobierno central (viceministros), un } \\
\text { exsecretario general autonómico, un } \\
\text { director general del Gobierno central y } \\
\text { dos directores generales de Comunidades } \\
\text { Autónomas }\end{array}$ & 63,7 \\
\hline Profesores de Universidad & $\begin{array}{l}\text { Siete profesores universitarios con amplia } \\
\text { experiencia docente e investigadora de } \\
\text { cinco universidades españolas }\end{array}$ & 56,1 \\
\hline $\begin{array}{l}\text { Directores o exdirectores de } \\
\text { Instituto de Educación Se- } \\
\text { cundaria }\end{array}$ & $\begin{array}{l}\text { Cuatro directores en ejercicio y tres } \\
\text { exdirectores, de cuatro Comunidades } \\
\text { Autónomas, con amplia experiencia } \\
\text { tanto en el ámbito de la acción como en } \\
\text { el de la reflexión sobre la práctica de la } \\
\text { dirección escolar }\end{array}$ & 58,3 \\
\hline
\end{tabular}

Fuente: Elaboración propia.

\subsubsection{La información inicial}

De forma previa al desarrollo de la ronda 1 , se ha puesto a disposición de los miembros del panel toda la documentación necesaria para la participación en el estudio:

- Una breve descripción de la técnica Delphi.

- Información procedente de los coordinadores del estudio y relativa a las bases conceptuales del conjunto de dimensiones y subdimensiones del sistema indicadores previamente definido.

- Los criterios de selección del panel.

- El cuestionario digital con espacios para los comentarios y las reflexiones, con la posibilidad de añadir nuevos indicadores.

- Unas breves pero claras indicaciones de uso.

\subsubsection{El feedback}

El feedback a los miembros del panel, al terminar cada ronda, ha sido de tipo tanto cualitativo como cuantitativo. El "feedback cualitativo" ha supuesto el envío a todos los expertos, tras cada ronda, de un resumen común, en forma anónima, de los comentarios efectuados por ellos mismos y, en algún caso, por los coordinadores del estudio. Ello ha permitido corregir ítems o incluso incorporar al cuestionario otros nuevos, así como evidenciar posiciones individuales ante el grupo de los distintos expertos con vistas a la ronda siguiente para aquellos ítems objeto de valoración. El "feedback cuantitativo" ha consistido en proporcionar, para cada ítem de la versión del cuestionario correspondiente a cada ronda, dos parámetros -la media (M) y 
el coeficiente de variación intercuartil (CVI)- que reflejan el grado de importancia y el nivel de consenso, respectivamente, y expresan numéricamente la opinión del grupo en cada ronda para cada uno de los ítems sometidos a la valoración de los expertos del panel.

\subsubsection{Los niveles de importancia}

Se han calculado los niveles de importancia atribuibles a las diferentes categorías y subcategorías, a partir de los resultados definitivos mediante los valores de las medias de acuerdo con la escala de Likert empleada [nada importante (0), poco importante (1), relativamente importante (2), muy importante (3), extremadamente importante (4)].

\subsubsection{El grado de consenso}

Se ha elegido como parámetro que expresa el grado de convergencia o de consenso entre las puntuaciones de los expertos el Coeficiente de Variación Intercuartil (CVI) (Bonett, 2006; Ngoc Quyen, 2014), definido como: $C V I=(Q 3-Q 1) /(Q 3+Q 1)$, donde $Q 1$ se corresponde con el percentil 25 y $\mathrm{Q} 3$ con el percentil 75.

En cuanto al parámetro de estabilidad, que refleja la no variación significativa de la opinión de los expertos entre dos rondas sucesivas, se ha ignorado en este caso, de acuerdo con el criterio de Landeta (1999), según el cual "si hay consenso debe haber estabilidad, pero la existencia de estabilidad no implica que se haya alcanzado el consenso" (p. 91).

\subsubsection{El número de rondas realizadas}

El número de rondas se ha determinado de modo que se lograran niveles satisfactorios tanto de importancia como de consenso. Así, se ha fijado $\mathrm{M}=3$ como el valor umbral por encima del cual se considera suficiente la valoración de la importancia del ítem $(0 \leq \mathrm{M} \leq 4)$; y $\mathrm{CVI}=0,2$ como el valor umbral por debajo del cual se considera que existe consenso $(0 \leq \mathrm{CVI} \leq 1)$.

Los indicadores que en cualquiera de las rondas lograron ambos estándares fueron dados por buenos y apartados del proceso de valoración en la siguiente, salvo en aquellos casos en los cuales las recomendaciones de los expertos suponían la modificación del ítem y la introducción de algún cambio sustantivo en la redacción; en tales supuestos la nueva redacción fue sometida a valoración en la ronda siguiente. Por otra parte, aquellos ítems que, bien en su redacción inicial o en su versión enriquecida, no consiguieron alcanzar ambos estándares de importancia y de consenso fueron excluidos del sistema. La aplicación de tales criterios ha llevado a la aplicación de tres rondas de consultas al panel de expertos. 


\section{Resultados}

\subsection{Evolución cuantitativa del proceso DELPHI}

En la primera ronda, no fue rechazado ninguno de los 66 ítems que configuraban la versión cero, o inicial, del cuestionario, lo que refuerza la validez del punto de partida. No obstante, 39 requirieron, a juicio de algún miembro del panel, pequeñas mejoras en la redacción, 6 fueron aceptados con cambios sustantivos y valorados en la siguiente ronda y fueron añadidos 38 nuevos ítems, aunque sin alterar el marco de categorías y de subcategorías previamente establecido. A este respecto, cabe aclarar que los miembros del panel tuvieron ocasión de hacerlo en esta primera ronda.

En la segunda ronda, de los 104 ítems que constituían la nueva versión del cuestionario (versión 1 ), 30 requirieron, a juicio de algún miembro del panel, pequeñas mejoras en la redacción y otros 40 se sumaron a los 20 de la ronda anterior y no requirieron valoración por parte de los expertos en la ronda siguiente. Sin embargo, 1 ítem supuso modificaciones que sí requirieron una nueva valoración por parte del panel, 12 ítems fueron rechazados de entre las nuevas aportaciones y fueron aceptados 2 nuevos más.

En la tercera ronda, los 93 ítems de la ronda anterior (versión 2 del cuestionario) fueron aceptados por haber alcanzado los umbrales de importancia y de consenso previamente definidos. No obstante, un análisis pormenorizado de redundancias, efectuado por el equipo coordinador, llevo consigo la eliminación de otros 5 ítems, quedando el cuestionario, en su versión final (versión 3), compuesto por 88 ítems cuyo detalle se presenta en el anexo. La tabla 5 muestra un resumen cuantitativo de la evolución del cuestionario a lo largo de las tres rondas que configuraron el proceso Delphi.

\subsection{Niveles de importancia, pesos y grados de consenso}

En la tabla 6 se muestran los valores de M. Tanto en el caso de las dimensiones como en el de las subdimensiones, el valor de $\mathrm{M}$ recoge el promedio ${ }^{2}$ de las valoraciones simples (ítems) según la escala de Likert antes referida. Todos los valores sobrepasan, obviamente, el valor umbral fijado en 3 puntos, pero destacan con valores medios iguales o superiores a 3,5-sobre un máximo de 4 puntos- 8 de las 20 subcategorías (2.2, 2.3, 3.1, 4.2, 4.4, $5.1,6.1$ y 6.2 ) y 4 de las 6 categorías $(2,3,5$ y 6 ) (Tabla 6 ).

\footnotetext{
5 niveles de respuesta.
}

2 Se ha optado por la media al contar con una variable cuasi-cuantitativa con 
Tabla 5. Resumen cuantitativo de la evolución del proceso Delphi

\begin{tabular}{|c|c|c|c|c|c|}
\hline & $\begin{array}{l}\text { Versión } \\
\text { inicial }\end{array}$ & Ronda 1 & Ronda 2 & Ronda 3 & $\begin{array}{l}\text { Versión } \\
\text { final }\end{array}$ \\
\hline $\begin{array}{l}\text { Número de ítems aceptados } \\
\text { sin cambios en su redacción }\end{array}$ & & 20 & 60 & 90 & 93 \\
\hline $\begin{array}{l}\text { Número de ítems acepta- } \\
\text { dos con ligeros cambios en } \\
\text { su redacción que no requie- } \\
\text { ren una nueva valoración }\end{array}$ & & 39 & 30 & 0 & \\
\hline $\begin{array}{l}\text { Número de ítems acepta- } \\
\text { dos con cambios sustan- } \\
\text { tivos en su redacción que } \\
\text { sí requieren una nueva } \\
\text { valoración }\end{array}$ & & 6 & 1 & 3 & \\
\hline $\begin{array}{l}\text { Número de ítems que se han } \\
\text { cambiado de ubicación con } \\
\text { relación a las subcategorías }\end{array}$ & & 1 & 0 & 0 & \\
\hline Número de ítems rechazados & & 0 & (12) & 0 & (5) \\
\hline $\begin{array}{l}\text { Número de ítems completa- } \\
\text { mente nuevos }\end{array}$ & & 38 & 2 & 0 & \\
\hline $\begin{array}{l}\text { Total del número de ítems } \\
\text { del cuestionario }\end{array}$ & 66 & 104 & 93 & 93 & 88 \\
\hline
\end{tabular}

Fuente: Elaboración propia.

Tabla 6. Niveles de importancia, pesos y grados de consenso para los indicadores de calidad de la gobernanza del sistema educativo establecidos por el grupo de expertos, agrupados por dimensiones y subdimensiones

\begin{tabular}{lccc}
\hline & $\begin{array}{c}\text { Importancia } \\
\text { (M, de 0 a 4) }\end{array}$ & $\begin{array}{c}\text { Peso } \\
(\mathrm{P}, \%)\end{array}$ & $\begin{array}{c}\text { Consenso } \\
\text { (CVI, de 0 a 1) }\end{array}$ \\
\hline $\begin{array}{l}\text { 1. Gobernanza con un enfoque holístico y multinivel } \\
\text { 1.1. Toma en consideración la existencia de relaciones }\end{array}$ & $16,2 \%$ & 0,14 \\
\hline $\begin{array}{l}\text { e interdependencias entre los diferentes niveles del } \\
\text { sistema (individual, escolar y gubernamental) }\end{array}$ & 3,34 & $26,0 \%$ & 0,14 \\
\hline $\begin{array}{l}\text { 1.2. Preserva la importancia del nivel estatal } \\
\text { 1.3. Busca el consenso posible }\end{array}$ & 3,38 & $21,0 \%$ & 0,14 \\
\hline $\begin{array}{l}\text { 1.4. Promueve la participación de los actores de niveles } \\
\text { inferiores, principalmente del personal docente }\end{array}$ & 3,37 & $31,5 \%$ & 0,14 \\
\hline $\begin{array}{l}\text { 2. Gobernanza con capacidad de dirección } \\
\text { 2.1. Dispone de una visión estratégica }\end{array}$ & 3,52 & $16,8 \%$ & 0,12 \\
\hline $\begin{array}{l}\text { 2.2. Establece con claridad las prioridades } \\
\text { 2.3. Concede mucha importancia a la selección de los }\end{array}$ & 3,70 & $17,5 \%$ & 0,05 \\
\hline $\begin{array}{l}\text { responsables de las políticas } \\
\text { 2.4. Comporta una fuerte implicación personal de los } \\
\text { responsables de las políticas con los procesos de mejora }\end{array}$ & 3,48 & $22,0 \%$ & 0,14 \\
\hline
\end{tabular}




\begin{tabular}{|c|c|c|c|}
\hline & $\begin{array}{l}\text { Importancia } \\
(\mathrm{M}, \text { de } 0 \text { a } 4)\end{array}$ & $\begin{array}{l}\text { Peso } \\
(\mathrm{P}, \%)\end{array}$ & $\begin{array}{l}\text { Consenso } \\
\text { (CVI, de } 0 \text { a } 1)\end{array}$ \\
\hline 3. Gobernanza centrada en los procesos & 3,50 & $16,7 \%$ & 0,12 \\
\hline 3.1. Ejerce correctamente sus funciones principales & 3,63 & $33,0 \%$ & 0,07 \\
\hline 3.2. Realiza una gestión eficiente de los recursos & 3,40 & $26,5 \%$ & 0,14 \\
\hline 3.3. Elabora una regulación apropiada y de calidad & 3,44 & $22,4 \%$ & 0,14 \\
\hline 3.4 Otorga mucha importancia a la implementación & 3,48 & $18,1 \%$ & 0,14 \\
\hline $\begin{array}{l}\text { 4. Gobernanza basada en el conocimiento, la evidencia } \\
\text { empírica y la investigación }\end{array}$ & 3,42 & $16,3 \%$ & 0,14 \\
\hline $\begin{array}{l}\text { 4.1. Promueve y emplea el conocimiento y la inves- } \\
\text { tigación para una mejor formulación de las políticas }\end{array}$ & 3,33 & $30,0 \%$ & 0,14 \\
\hline 4.2. Es flexible y adaptativa & 3,50 & $23,6 \%$ & 0,14 \\
\hline 4.3. Otorga mucha importancia al feedback & 3,33 & $22,5 \%$ & 0,14 \\
\hline 4.4. Evalúa el impacto de las políticas & 3,53 & $23,9 \%$ & 0,14 \\
\hline 5. Gobernanza basada en la construcción de capacidades & 3,50 & $16,7 \%$ & 0,14 \\
\hline $\begin{array}{l}\text { 5.1. Establece diferentes fórmulas para compartir } \\
\text { con los actores principales el conocimiento sobre las } \\
\text { políticas y sobre su implementación }\end{array}$ & 3,58 & $58,4 \%$ & 0,14 \\
\hline $\begin{array}{l}\text { 5.2. Ayuda a los actores principales a adquirir y a } \\
\text { utilizar información relevante, a su nivel, para el éxito } \\
\text { en la implementación de las políticas }\end{array}$ & 3,40 & $41,6 \%$ & 0,14 \\
\hline 6. Gobernanza vinculada a la rendición de cuentas & 3,60 & $17,2 \%$ & 0,12 \\
\hline 6.1. Concede una gran importancia a la transparencia & 3,53 & $43,5 \%$ & 0,14 \\
\hline 6.2. Establece mecanismos de responsabilidad & 3,66 & $56,5 \%$ & 0,09 \\
\hline
\end{tabular}

Fuente: Elaboración propia.

En lo que concierne a los pesos, que se muestran también en la misma tabla, se expresan en términos relativos o de porcentajes de suma 100 para el conjunto de las subdimensiones de una dimensión dada, y también para el conjunto de las seis dimensiones o categorías establecidas. Por su propia definición, dan cuenta de la importancia relativa que el panel de expertos ha atribuido a las diferentes subdimensiones dentro de una dimensión dada, y a cada una de esas seis dimensiones constitutivas del modelo de evaluación que se deriva del marco conceptual.

Cabe destacar la relativa homogeneidad de los pesos de las categorías, pues se distancian poco del valor $16,7 \%$ que supondría la total igualdad. No obstante, destaca a este respecto la categoría 6 "Gobernanza vinculada a la rendición de cuentas", con un peso de 17,2\%. 
En cuanto a las subcategorías, y a la vista del análisis de los pesos, el juicio de los expertos ha situado en la cúspide de los indicadores o criterios de calidad de la gobernanza de un sistema educativo moderno -con el peso máximo dentro de cada subcategoría- los siguientes rasgos característicos:

- 1.4. Promueve la participación.

- 2.2. Establece con claridad las prioridades.

- 3.1. Ejerce correctamente sus funciones principales.

- 4.1. Promueve y emplea el conocimiento y la investigación para una mejor formulación de las políticas.

- 5.1. Establece diferentes fórmulas para compartir con los actores principales el conocimiento sobre las políticas y sobre su implementación.

- 6.2. Establece mecanismos de responsabilidad.

En la tabla 6 se presentan, asimismo, los niveles finales de consenso medido a través del CVI, indicador que es tanto menor cuanto mayor es aquel. Aun cuando todas las subcategorías presentan un grado de consenso promedio elevado -como corresponde al proceso Delphi previo al que fue sometido el cuestionario- destacan, en particular, las subcategorías: "2.3. Concede mucha importancia a la selección de los responsables de las políticas" (CVI =0,05), "3.1. Ejerce correctamente sus funciones principales" $(\mathrm{CVI}=0,07)$ y "6.2. Establece mecanismos de responsabilidad" (CVI =0,09).

Si se incrementa aún más el nivel de exigencia en la selección de criterios y se exige de las subcategorías, no sólo un elevado peso sino también un muy alto consenso $(\mathrm{CVI}<0,1)$, resulta esta terna de rasgos prioritarios:

- 2.3. Concede mucha importancia a la selección de los responsables de las políticas.

- 3.1. Ejerce correctamente sus funciones principales.

- 6.2. Establece mecanismos de responsabilidad.

De este primer análisis deriva un código de conducta para los responsables políticos -de carácter normativo- sencillo en su formulación, pero de apreciable interés para mejorar la calidad de la gobernanza de los sistemas educativos. 


\subsection{Fiabilidad y validez del instrumento}

\subsubsection{Fiabilidad}

Un estudio exploratorio posterior sobre la calidad de la gobernanza del sistema educativo español realizado por el panel de expertos, utilizando el instrumento de diagnóstico antes descrito, ha permitido entre otras cosas evaluar su fiabilidad (consistencia interna), medida mediante el alfa de Cronbach, para cada una de sus seis dimensiones. La tabla 7 muestra los resultados correspondientes. Como puede apreciarse en dicha tabla, nos encontramos ante extraordinarios valores de fiabilidad en el análisis desagregado por dimensiones, ya que los valores del estadístico alfa de Cronbach oscilan entre un 0,92, obtenido para la dimensión 5, y un 0,98 para la dimensión 3 .

Tabla 7. Análisis de fiabilidad (consistencia interna) del instrumento de evaluación para cada una de las seis dimensiones que lo configuran

\begin{tabular}{lcc}
\hline & $\begin{array}{c}\text { Alfa de } \\
\text { Cronbach }\end{array}$ & $\begin{array}{c}\text { Número de } \\
\text { elementos }\end{array}$ \\
\hline Dimensión 1. Gobernanza con un enfoque holístico multinivel & 0,963 & 19 \\
\hline Dimensión 2. Gobernanza con capacidad de dirección & 0,974 & 18 \\
\hline Dimensión 3. Gobernanza centrada en los procesos & 0,978 & 22 \\
\hline $\begin{array}{l}\text { Dimensión 4. Gobernanza basada en el conocimiento, la evidencia } \\
\text { empírica y la investigación }\end{array}$ & 0,965 & 13 \\
\hline Dimensión 5. Gobernanza basada en la construcción de capacidades & 0,933 & 7 \\
\hline Dimensión 6. Gobernanza vinculada a la rendición de cuentas & 0,959 & 9 \\
\hline
\end{tabular}

Fuente: Elaboración propia.

\subsubsection{Validez}

En el contexto conceptual de la evaluación, el término validez hace referencia a la conveniencia y utilidad de una prueba para un propósito particular. Entre las cinco formas de evidencias de validez establecidas por los Standards for Educational and Psychological Testing de la American Educational Research Association (2014), se encuentra la evidencia de validez basada en el contenido del test. Los resultados analizados anteriormente, así como la conceptualización previa, aportan evidencias de validez de contenido suficientes como para amparar la adecuación del instrumento propuesto a la finalidad que se pretende. 


\section{Discusión}

La aplicación del método Delphi en la elaboración de un cuestionario para la evaluación de sistemas educativos ha tenido un triple propósito: validación del marco conceptual, refinamiento del correspondiente cuestionario y enriquecimiento del mismo.

A pesar de que el panel de expertos tenía la posibilidad de añadir ítems correspondientes a subdimensiones o incluso a dimensiones de la calidad de la gobernanza educativa, complementarias a las previamente definidas, Io cierto es los 40 ítems nuevos aportados por el panel en el proceso Delphi estuvieron enmarcados todos ellos en esa estructura conceptual inicial de conjunto. Ello proporciona una validación empírica del marco que va más allá de la que se deriva de su propio método de construcción y por tanto la refuerza. Resulta coherente con lo anterior el hecho de que el $40 \%$ de las subcategorías y el $66,7 \%$ de las categorías hayan alcanzado un valor de $\mathrm{M}$ igual superior a 3,5 puntos sobre 4 .

Cabe, asimismo, señalar la intensidad de la participación de los expertos en el proceso de consulta. Ello se ha reflejado en la producción de un total de 258 comentarios a lo largo de las tres rondas del proceso Delphi (80 comentarios en la primera ronda, 155 en la segunda ronda y 23 comentarios en la tercera ronda). Además, y sobre una cifra de partida de 66 ítems, el panel ha aportado otros 40 más. De ese volumen total de 106 ítems han resistido, finalmente, el proceso iterativo de selección 88. A la vista de estos datos, cabe afirmar que se han hecho efectivos tanto los mecanismos de refinamiento, propios del Delphi, como los de enriquecimiento del cuestionario, lo cual resulta coherente con la elevada competencia profesional de los 21 componentes del panel de expertos y con un grado de implicación personal en el proceso no inferior.

Más allá de los anteriores subrayados, procede destacar la decantación de todo el esfuerzo analítico en una selección, efectuada sobre una base empírica, de seis subdimensiones que aluden a otros tantos rasgos fundamentales de una buena gobernanza en el nivel macro de los sistemas educativos. A continuación, se enuncian esos seis rasgos reordenados en un orden conceptual de generalidad decreciente (Tabla 6).

El rasgo (1) "Ejerce correctamente sus funciones principales" (subdimensión 3.1) posee un alto nivel de generalidad que, aun a pesar de haber sido definido en el proceso de elaboración de un modo independiente al de los seis rasgos subsiguientes, converge con ellos en el sentido de que 
éstos pueden ser considerados como concreciones de aquel. La valoración de los expertos ha permitido, al parecer, alumbrar un constructo con una elevada coherencia interna.

Aun cuando, en la práctica, sea ignorado frecuentemente como rasgo esencial de una gobernanza de calidad de los sistemas educativos, un enfoque basado en prioridades -rasgo (2) "Establece con claridad las prioridades" (subdimensión 2.2) - constituye una cuestión esencial para todo tipo de organizaciones y, en particular, para la gestión de los sistemas organizacionales altamente complejos como lo son los sistemas de educación y de formación. Pero no sólo se trata de adoptar esta clase de enfoques, sino de hacerlo del modo más efectivo posible. Y para conducirse con alguna garantía de éxito en este tipo de entornos conviene tomar en consideración la advertencia, atribuida a Jacques Delors, según la cual "no es posible tener ciento cincuenta prioridades" 3 .

En este punto, merece la pena traer a colación el Ilamado "principio de Pareto", o "ley universal de las prioridades", que en su versión original -en cuanto a la eficacia de los factores que operan sobre sistemas sociales complejos- establece: "Pocos vitales, muchos triviales". Esa formulación del sociólogo y economista italiano Wilfredo Pareto (1848-1923), fue reformulada por Joseph Juran (1904-2008) en la década de los cincuenta del pasado siglo y aplicada al mundo de la calidad en la gestión de las organizaciones bajo la conocida "regla 20-80". Dicha regla, de naturaleza empírica, sostiene que el 20 por ciento de las causas, inputs o esfuerzos explica el 80 por ciento de los efectos, outputs o recompensas (Koch, 1998).

Aplicado lo anterior a la gobernanza de los sistemas educativos, no se trata de dispersar los esfuerzos en mil y una medidas cuyo impacto real sobre los outputs del sistema es pequeño, o incluso insignificante, sino de identificar primero ese grupo relativamente reducido de factores críticos, cuyo impacto sobre la mejora de los resultados escolares es máximo, y, a continuación, centrar sobre él las políticas educativas correspondientes.

Es claro que, en la gobernanza de los sistemas educativos, resulta imprescindible dejar a un lado las orientaciones cortoplacistas, los planteamientos oportunistas y las posiciones corporativistas en beneficio de los intereses generales. Y en esta tarea, el rasgo (4) "Promueve y emplea el conocimiento y la investigación para una mejor formulación de las políticas" (subdimensión 4.1) viene al auxilio del (2) "Establece con claridad las prioridades" para hacerlo más efectivo.

${ }^{3}$ Comentario oral de Felipe González Márquez, expresidente del gobierno español. 
A lo largo de las últimas tres décadas se ha ido generando, en el ámbito internacional, bastante evidencia empírica sobre el impacto de las políticas y los factores que podrían explicar, en mayor medida, los buenos resultados escolares de los alumnos. Pero quizás sea el trabajo de meta-síntesis de John Hattie (2008) -efectuado sobre más de 800 meta-análisis- el más ambicioso a la hora de identificar factores asociados, en un mayor grado, con las cifras de rendimiento escolar o que expliquen su varianza. Otros metaanálisis amplios, centrados en grupos concretos de políticas, están asimismo disponibles (Robinson 2009; López López 2014; Castro et al. 2015). Sin ignorar las limitaciones que presentan los enfoques correlacionales a la hora de establecer, sin ambigüedad, relaciones causales (Pawson, 2006), se trata de que los responsables políticos aseguren una aplicación ordenada y sistemática del antes referido rasgo (4). Algunos países, como es el caso de Nueva Zelanda a través de su proyecto BES (Best Evidence Synthesis), hace al menos una década que iniciaron este camino. Ese movimiento de globalización de las políticas, antes referido, no resulta independiente del proceso internacional acelerado de generación de evidencias (Hallinger, 2014).

El rasgo (3) "Establece mecanismos de responsabilidad" (subdimensión 6.2) es complementado por la subdimensión 2.3 "Concede mucha importancia a la selección de los responsables de las políticas", subdimensión que goza de un elevado consenso. Los mecanismos de selección de los responsables políticos constituyen la conditio sine qua non de la calidad de la gobernanza, en general, y de la educativa en particular. Sin embargo, la evolución de la dinámica de los partidos políticos en los países desarrollados, por un lado, y la proliferación de niveles de decisión asociados a los frecuentes procesos de descentralización política y administrativa, por otro, están Ilevando consigo, en algunos casos, una relajación extrema en cuanto a las exigencias de idoneidad para cubrir los puestos decisorios en materia de gobernanza. Por lo general, el incumplimiento de este criterio de calidad de la gobernanza suele acompañarse de una falta de interés por los mecanismos de responsabilidad, o de rendición de cuentas, por los resultados obtenidos. De este modo, los déficits en cuanto al primero de estos dos criterios se ven amplificados por los efectos deletéreos del incumplimiento del segundo, con lo cual los sistemas educativos corren el riesgo de acomodarse a lo que se conoce como "calidad de supervivencia", esto es, la calidad mínima imprescindible para asegurar la supervivencia individual o social.

Finalmente, los rasgos (5) "Promueve la participación" (subdimensión 1.4) y (6) "Establece diferentes fórmulas para compartir con los actores principales el conocimiento sobre las políticas y sobre su implementación" (subdimensión 5.1) convergen con ese hallazgo feliz de Ben Levin (2010) que concluye en que las reformas educativas no podrán tener éxito sin com- 
prometer la mente de los protagonistas y sus corazones. Esa conclusión de Levin, -en particular, la dimensión emocional del liderazgo de las reformas educativas-, basada en una amplia experiencia, está teniendo una apoyatura empírica en la investigación básica de naturaleza neurocientífica sobre el liderazgo de las personas en el seno de las organizaciones, o "liderazgo transformacional” (Wang, 2019). La participación de los actores, si es conducida de una forma adecuada, estimulará la adhesión a las reformas educativas de buena parte de ellos, actuando primordialmente -aunque no sólo- sobre sus "corazones". Por otro lado, el hecho de compartir el conocimiento experto, en el que se apoyan las reformas, con los destinatarios de las mismas es una manera de, operando sobre sus mentes, promover su alineamiento con los objetivos de dichas reformas y con las correspondientes políticas. No obstante lo anterior, esta distinción nítida entre emoción y cognición sabemos que es tan sólo una ilusión analítica, pues nuestro cerebro las integra en unos bucles causales de interacción que las debilitan o fortalecen recíprocamente, tal y como la neurociencia se ha encargado de evidenciar (Hinton et al., 2008; Fuster, 2012, 2013).

\section{Referencias}

American Psychological Association, National Council on Measurement in Education, \& American Educational Research Association (2014). Standards for educational and psychological testing. Washington, DC: American Educational Research Association.

Bonett, D. G. (2006). Confidence interval for a coefficient of quartile variation". Computacional Statistics and Data Analysis, 50(11), 2953-2957. https://doi.org/10.1016/j. csda.2005.05.00.

Boulkedid, R., Abdoul, H., Loustau M., Sibony, O. \& Alberti, C. (2011). Using and reporting the Delphi Method for Selecting Healthcare Quality Indicators: A Systematic Review. PLOS ONE 6(6) e 20476. https://doi.org/10.1371/journal.pone.0020476.

Castro, M., Expósito-Casas, E., López-Martín, E., Lizasoain, L., Navarro-Asencio, E. \& Gaviria, J. L. (2015). Parental involvement on student academic achievement: A meta-analysis. Educational Research Review, 14, 33-46.

Fazekas, M. \&Tracey, B. (2012). Exploring the Complex Interaction Between Governance and knowledge in Education. OECD Education Working Papers, 67, OECD Publishing. Doi: $10.1787 / 5 \mathrm{k} 9 \mathrm{flc} 2 \mathrm{I} 340$-en

Fuster, J. (2012). “Entrevista”. Participación educativa. Segunda época, 1(1), 29-31. Recuperado de https://bit.ly/2x96IDX

Fuster, J. (2013). The neuroscience of freedom and creativity. Our predictive brain. Padstow, Cornwall: Cambridge University Press.

Hallinger, P. (2014). Reviewing reviews of research in education leadership: An empirical assessment. Educational Administration Quaterly,50(4), 539-576. First published date: October-23-2013. https://doi.org/10.1177/0013161X13506594. 
Hanushek, E. A., Rivkin, S.G. \& Schiman, J. C. (2016). Dynamic effects of teacher turnover on the quality of instruction. Economics of Education Review, 55,132-148.

Hasson, F., Keeney, S. \& McKrenna, H. (2000). Research guidelines for the Delphi survey technique. J. Adv. Nurs. 32, 1008-1015.

Hattie, J. (2003). Teachers Make a Difference: What is the research evidence? Australian Council for Educational Research Annual Conference on: Building Teacher Quality. October 2003. pp 1-17. Recuperado de https://bit.ly/2Xftq2p

Hattie, J. (2008). Visible Learning: A Synthesis of Over 800 Meta-Analyses Relating to Achievement. Abingdon: Routledge.

Hattie, J. (2017). “Aprendizaje visible” para profesores. Maximizando el impacto del aprendizaje. Madrid: Paraninfo.

Hewitt de Alcántara, C. (1998). Uses and Abuses of the Concept of Governance. International Social. Science Journal, 50(1): 105-113.

Hinton, Ch., Miyamoto, K. \& della Chiesa, B. (2008): Brain Research, Learning and Emotions: implications for education research, policy and practice. European Journal of Education, 43(1), 87-103.

Kaufman, D., Kraay, A., \& Zoido-Lobatón, P. (2000). Governance matters: From measurement to action. Finance and Development, 37(2), 10-13.

Koch, R. (1998). The 80/20 Principle. The Secret of Achieving More with Less. London: Nicholas Brealey Publishing. Recuperado de https://bit.ly/2xU5PtC

Lamb, S., Maire, Q. \& Doecke, E. (2017). Key Skills for the 21st Century: an evidence-based review. Sidney: NSW Governement. Recuperado de https://bit.ly/2Vbgfg6

Landeta, J. (1999). El método Delphi. Una técnica de previsión para la incertidumbre. Barcelona: Editorial Ariel.

Leithwood, K., Seashore,K., Anderson, S. \& Wahlstrom, K. (2004). How leadership influences student learning. New York: The Wallace Foundation. Recuperado de https://bit. ly/2XkcUhB

Leithwood, K. \& Louis, K.S. (2012). Linking leadership to student learning. San Francisco: Jossey-Bass.

Levin, B. (2010). Governments and education reforms: some lessons from the last 50 years. Journal of Education Policy, 25(6), 737-747.

Linstone, H. A. \& Turroff, M. (2002). The Delphi method: Techniques and Applications. H. A. Linstone (Ed.). Recuperado de https://bit.ly/2JIJtxB.

López López, E. (2014). El mastery learning a la luz de la investigación educativa. Participación Educativa, segunda época, 3(5), Recuperado de https://bit.ly/3cOGHzQ.

López Rupérez, F. (1997). Complejidad y Educación. Revista Española de Pedagogía, 206, 103-112

López Rupérez, F. (2001). Preparar el futuro. La educación ante los desafíos de la globalización. La Muralla. Madrid. 
López Rupérez, F., García García, I. \& Expósito Casas, E. (2017). La calidad de la gobernanza del sistema educativo español. Un estudio empírico. Universidad Camilo José Cela. Madrid. Recuperado de https://bit.ly/2RfmgqX

López Rupérez, F., García García, I. \& Expósito Casas, E. (2020). School Leadership in Spain. Evidence from PISA 2015 assessment and Recommendations. Leadership and Policy in School. (en prensa).

Ngoc Quyen, D. T. (2014). Developing university governance indicators and their weighting system using a modified Delphi method. Procedia Social and Behavioral Sciences, 141, 828-833. https://doi.org/10.1016/j. sbspro.2014.05.144.

OCDE (2015). Política educativa en perspectiva. Hacer posibles las reformas. OCDE-Santillana. Madrid.

OECD (2016). Governing Education in a Complex World. Educational Research and Innovation, OECD Publishing: Paris.

OECD/CERI (2015). Governing Complex Education Systems (GCES). Recuperado de https:// bit.ly/2V3vyYo.

Pont Ferrer, B. (2017). Reformas educativas: el caso del liderazgo escolar en perspectiva comparada. Tesis Doctoral. Universidad Complutense de Madrid. Madrid.

Robinson, V., Hohepa, M. \& Lloyd, C. (2009). School Leadership and Student Outcomes: Identifying What Works and Why. Best Evidence Synthesis (BES). Ministry of Education \& University of Auckland. New Zealand. Recuperado de https://bit.ly/34f55eG.

Pawson, R. (2006). Evidence-Based Policy: A Realist Perspective. London, Thousand Oaks, New Delhi: SAGE Publications.

Schleicher, A. (2018). Primera Clase: Cómo construir una escuela de calidad para el siglo XXI, Paris: OECD Publishing / Madrid: Fundación Santillana. Recuperado de https://doi. org/10.1787/9788468050126-es.

United Nations (2007). Public Governance Indicators: A Literature Review. Department of Economics and Social affairs. Nueva York.

Uphoff, E., Wennekes, L., Grol. R., Wollersheim, H., Hermens, R. \& Ottevanger, P. (2012). Development of Generic Quality Indicators for Patient-Centered Cancer Care by Using a RAND Modified Delphi Method. Cancer Nursing ${ }^{T M}$, 35(1), 29-37. doi: 10.1097/ NCC.Ob013e318210e3a2.

Varela-Ruiz, M., Díaz-Bravo, L. \& García-Durán, R. (2012). Descripción y usos del método Delphi en investigaciones del área de la salud. Investigación en Educación Médica, 1(2): 90-95.

Wang, Y. (2019). Pulling at Your Heartstrings: Examining Four Leadership Approaches from the Neuroscience Perspective. Educational Administration Quarterly, 55(2), 328-359. https://doi.org/10.1177/0013161X18799471

World Bank (1994). Governance. The World Bank's Experience. Washington, D.C. 


\section{Anexo. Instrumento para una evaluación de la calidad de la gobernanza de los sistemas educativos}

Valore el nivel de calidad de la gobernanza del sistema educativo español, a través de cada uno de los diferentes criterios que se formulan a continuación, utilizando para ello la siguiente escala: [(0) nulo, (1) escaso, (2) mediano, (3) alto, (4) muy alto].

\begin{tabular}{|c|c|c|c|c|c|}
\hline \multicolumn{6}{|c|}{$\begin{array}{l}\text { 1.1 Toma en consideración la existencia de relaciones e interdependencias entre los diferentes niveles del sistema (individual, } \\
\text { escolar y gubernamental) }\end{array}$} \\
\hline $\begin{array}{l}\text { 1.1.1. Estudian la manera en que los comportamientos de los niveles inferiores pueden consolidar las } \\
\text { políticas definidas en los niveles superiores, y viceversa. }\end{array}$ & 0 & 1 & 2 & 3 & 4 \\
\hline $\begin{array}{l}\text { 1.1.2. Recogen de niveles inferiores información y se ocupan activamente de su satisfacción en el trabajo } \\
\text { de modo que repercuta en la mejora del conjunto del sistema. }\end{array}$ & 0 & 1 & 2 & 3 & 4 \\
\hline 1.1.3. Desarrollan actuaciones orientadas a promover beneficios recíprocos entre niveles. & 0 & 1 & 2 & 3 & 4 \\
\hline $\begin{array}{l}\text { 1.1.4. Establecen mecanismos que favorecen el diálogo entre los diferentes actores del sistema y promueven } \\
\text { un clima de confianza entre las partes. }\end{array}$ & 0 & 1 & 2 & 3 & 4 \\
\hline $\begin{array}{l}\text { 1.1.5. Toman en cuenta las relaciones entre el sistema educativo y su entorno, así como el impacto d } \\
\text { uno sobre el otro. }\end{array}$ & 0 & 1 & 2 & 3 & 4 \\
\hline
\end{tabular}

1.2. Preserva la importancia del nivel estatal

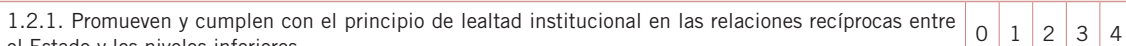
el Estado y los niveles inferiores.

\begin{tabular}{l} 
1.2.2. Desarrollan en los niveles superiores un liderazgo basado en la visión, en el conocimiento y en \\
\hline
\end{tabular} la confianza.

1.2.3. Cuidan las relaciones personales como factor que coadyuva a la consideración de la importancia de dicho nivel estatal.

1.2.4. Cumplen y hacen cumplir la normativa del nivel estatal.

1.3. Busca el consenso posible

1.3.1. Disponen de una visión clara del alcance del consenso, en el ámbito del sistema en su conjunto, pero también de sus limitaciones de cara a la mejora.

1.3.3. Manifiestan una objetividad sistemática en sus juicios y están abiertos a los criterios razonables de los otros.

$\begin{array}{llllll}0 & 1 & 2 & 3 & 4\end{array}$

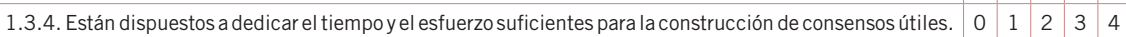

1.4. Promueve la participación de los actores de niveles inferiores, principalmente del personal docente

\begin{tabular}{|l|l|l|l|l|l|l|l|l} 
1.4.1. Se preocupan de que la información relevante para cada ámbito inferior de decisión llegue de un & 0 & 1 & 2 & 3 & 4
\end{tabular} modo directo y efectivo.

1.4.2. Aplican, en el ámbito de sus competencias, mecanismos sistemáticos de consulta a los directivos de centros educativos y a sus organizaciones profesionales.

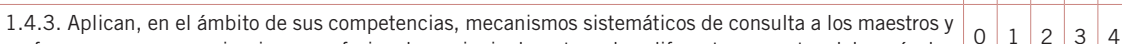
\begin{tabular}{lll|l|l|l|l|l} 
profesores y a sus organizaciones profesionales, principalmente, sobre diferentes aspectos del currículo. & 0 & 1 & 2 & 3 & 4
\end{tabular}

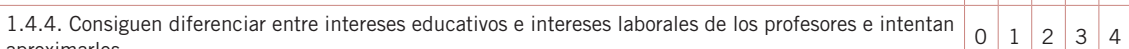
aproximarlos.

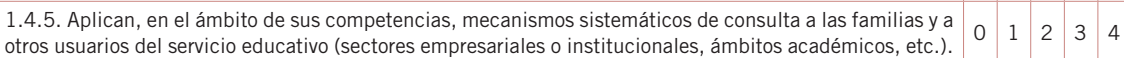

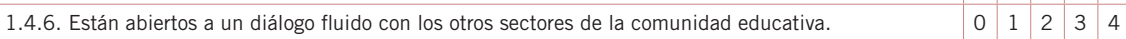

2. Gobernanza con capacidad de dirección

2.1. Dispone de una visión estratégica

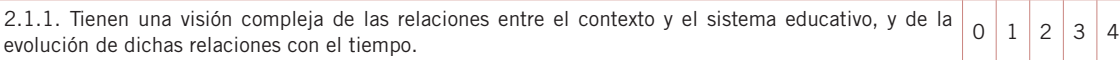

2.1.2. Integran la visión del sistema a largo plazo con una visión a medio y a corto plazo.

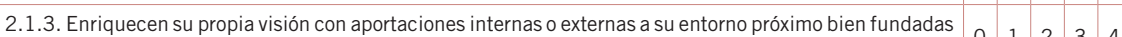
que contribuyan al acierto en su implementación.

\begin{tabular}{|l|l|l|l|l|l|l|l} 
2.1.4. Son capaces de anticiparse razonablemente al futuro mediante actuaciones que se apoyen en la & 0 & 1 & 2 & 3 & 4
\end{tabular} pertinencia y en la consistencia de la visión.

2.2. Establece con claridad las prioridades

\begin{tabular}{|l|l|l|l|l|l|l|l|l|l} 
2.2.1. Definen un sistema claro de prioridades para la mejora formado por un número relativamente & 0 & 1 & 2 & 3 & 4
\end{tabular} reducido de ellas.

2.2.2. Para la definición de las prioridades toman en consideración evidencias empíricas consolidadas relativas a su impacto sobre la realidad educativa. 
Valore el nivel de calidad de la gobernanza del sistema educativo español, a través de cada uno de los diferentes criterios que se formulan a continuación, utilizando para ello la siguiente escala: [(0) nulo, (1) escaso, (2) mediano, (3) alto, (4) muy alto]. \begin{tabular}{l}
$\begin{array}{l}\text { 2.2.3. Definen las prioridades para la mejora otorgando importancia al medio y largo plazos y no solo al } \\
\text { corto plazo. }\end{array}$ \\
\hline
\end{tabular}

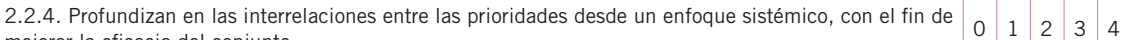
mejorar la eficacia del conjunto.

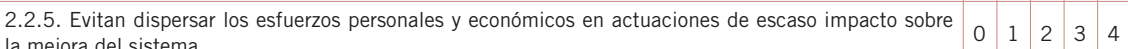
la mejora del sistema.

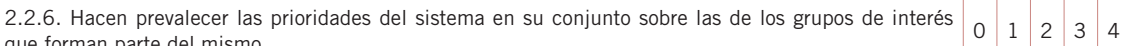
que forman parte del mismo.

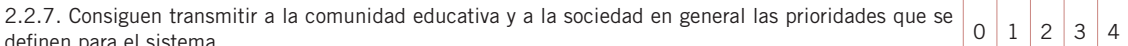
definen para el sistema.

\subsection{Concede mucha importancia a la selección de los responsables de las políticas}

\begin{tabular}{|l|l|l|l|l|l|l|l|l|l} 
2.3.1. Aplican con rigor los principios constitucionales de mérito y capacidad en la selección de respon- & 0 & 1 & 2 & 3 & 4
\end{tabular} sables de las políticas a un alto nivel.

2.3.2. Evitan que los compromisos políticos, institucionales o personales eleven a posiciones de responsa- $\left.{ }_{0}\right]_{2} 3_{3}$

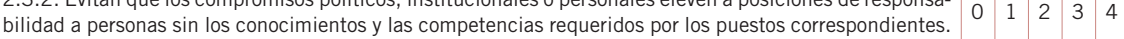

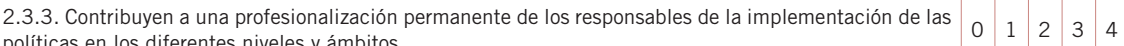
políticas en los diferentes niveles y ámbitos.

2.4. Comporta una fuerte implicación personal de los responsables de las políticas con los procesos de mejora

\begin{tabular}{|l|l|l|l|l|l|l|l|l} 
2.4.1. Demuestran, de un modo ostensible, a los actores de los niveles inferiores un compromiso personal & 0 & 1 & 2 & 3 & 4
\end{tabular} con el desarrollo acertado de las políticas.

2.4.2. Participan, a su nivel, en tareas de comunicación y de formación del resto de los actores principales desde la máxima disponibilidad personal posible.

2.4.3. Impulsan procesos de mejora, basados en la innovación y la autonomía, estableciendo mecanismos de evaluación y de rendición de cuentas.

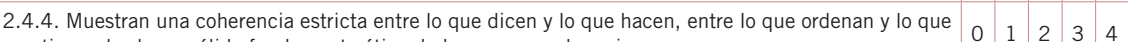
practican, desde un sólido fundamento ético de los procesos de mejora.

3.1. Ejerce correctamente sus funciones principales

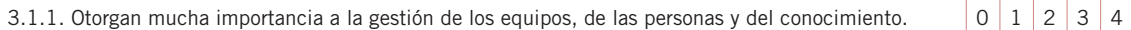

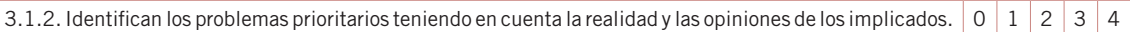

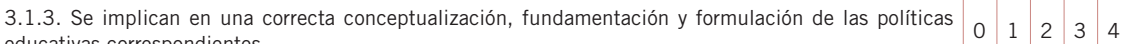
educativas correspondientes.

3.1.4. Promueven la evaluación de la calidad de las políticas desarrolladas, desde el punto de vista de los procesos que comportan, de los resultados que logran y del nivel de satisfacción de sus destinatarios.

3.1.5. Toman decisiones en función de los resultados obtenidos en la evaluación de la implementación de las políticas.

3.1.6. Promueven tanto la evaluación externa como la autoevaluación

\begin{tabular}{l|l|l|l|l|l}
0 & 0 & 2 & 3 & 4
\end{tabular}

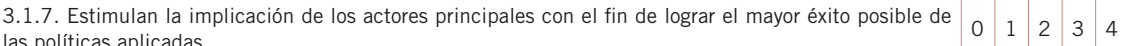
las políticas aplicadas.

3.2. Realiza una gestión eficiente de los recursos

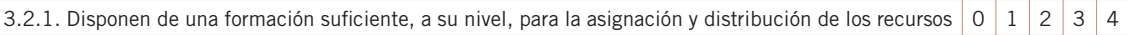

3.2.2. Poseen y utilizan mecanismos adecuados y rigurosos para determinar la cuantía de los recursos necesarios en la aplicación, con suficientes garantías, de las políticas previamente definidas.

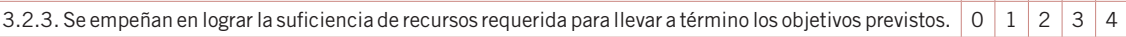

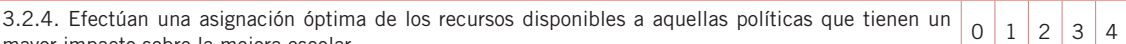
mayor impacto sobre la mejora escolar.

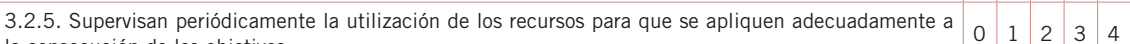
la consecución de los objetivos.

3.2.6. Mantienen en el tiempo la coherencia de las políticas y su financiación, evitando desviaciones por razones oportunistas o clientelares.

3.3. Elabora una regulación apropiada y de calidad

\begin{tabular}{ll|l|l|l|l|l|l|l|l} 
3.3.1. Vigilan para que el contenido de las normas se adecúe a las políticas que se pretenden regular, & 0 & 1 & 2 & 3 & 4
\end{tabular} sin distorsionarlas.

3.3.2. Cuidan la consistencia, la corrección y la asequibilidad de su formulación jurídica evitando redacciones oscuras y fallos o contradicciones, ya sean internos o con relación a otras normas. 
Valore el nivel de calidad de la gobernanza del sistema educativo español, a través de cada uno de los diferentes criterios que se formulan a continuación, utilizando para ello la siguiente escala: [(O) nulo, (1) escaso, (2) mediano, (3) alto, (4) muy alto]. \begin{tabular}{ll|l|l|l|l|l|} 
3.3.3. Procuran alinear con los objetivos de la normativa, mediante el diálogo, al mayor número posible & 0 & 1 & 2 & 3 & 4
\end{tabular} de actores relevantes del sistema educativo.

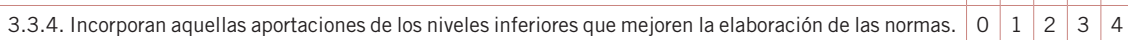

3.3.5. Promueven la simplificación burocrática en la concepción, aplicación y desarrollo de la normativa,

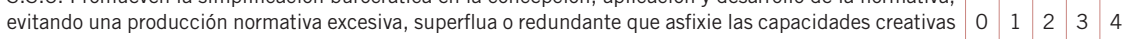
de los agentes del sistema.

3.4. Otorga mucha importancia a la implementación

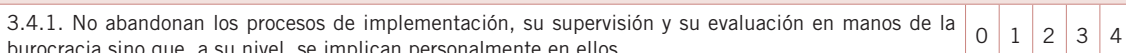
burocracia sino que, a su nivel, se implican personalmente en ellos. \begin{tabular}{l}
$\begin{array}{l}\text { 3.4.2. Implican a los niveles inferiores en la implementación de las políticas y procuran ganarse tanto } \\
\text { su mente como su corazón. }\end{array}$ \\
\hline
\end{tabular} \begin{tabular}{l|l|l|l|l|l|l}
$\begin{array}{l}\text { 3.4.3. Constituyen equipos de apoyo y organizan procedimientos para ayudar a los centros educativos en } \\
\text { los procesos de mejora, generando un clima de seguridad y de confianza. }\end{array}$ & 0 & 1 & 2 & 3 & 4
\end{tabular}

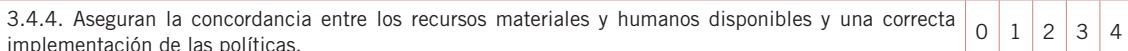
implementación de las políticas.

4. Gobernanza basada en el conocimiento, la evidencia empírica y la investigación

4.1. Promueve y emplea el conocimiento y la investigación para una mejor formulación de las políticas

\begin{tabular}{ll|l|l|l|l|l|l}
\hline 4.1.1. Disponen de una base de conocimiento experto actualizado y suficiente, a su nivel, que incluye el & 0 & 1 & 2 & 3 & 4
\end{tabular} que se deriva de informes internacionales.

4.1.2. Impulsan la realización de estudios y de revisiones para conocer el estado de la cuestión sobre

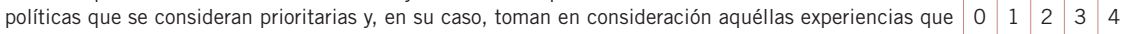
resultaron exitosas.

\begin{tabular}{ll|l|l|l|l|l}
\hline 4.1.3. Impulsan, en todos los niveles del sistema, investigaciones empíricas vinculadas a su mejora. & 0 & 1 & 2 & 3 & 4
\end{tabular} \begin{tabular}{ll|l|l|l|l|l|l}
\hline 4.1.4. Promueven actividades informativas y de formación para la difusión de políticas de éxito y de & 0 & 1 & 2 & 3 & 4
\end{tabular} buenas prácticas.

\subsection{Es flexible y adaptativa}

4.2.1. Se anticipan o reaccionan con relativa rapidez a los cambios que se advierten en el contexto general y que afectan o conciernen al sistema educativo en sus diferentes niveles.

\begin{tabular}{|l|l|l|l|l|l} 
4.2.2. Adaptan las políticas educativas a los diferentes contextos laborales, sociales, políticos y económi- & 0 & 1 & 2 & 3 & 4
\end{tabular} \begin{tabular}{ll|l|l|l|l}
$\cos$ a fin de conseguir resultados efectivos y satisfacer las expectativas razonables de sus destinatarios. & 0 & 1 & 2 & 3 & 4
\end{tabular} 4.2.3. Son capaces de conciliar el diálogo político y el acuerdo con otros actores, con la mejora de la \begin{tabular}{lll|l|l|l|}
\hline & 0 & 1 & 2 & 3 & 4
\end{tabular} calidad del sistema educativo y de sus resultados.

4.3. Otorga mucha importancia al feedback

\begin{tabular}{|l|l|l|l|l|l} 
4.3.1. Disponen de un sistema institucionalizado de generación de información que facilite el feedback. & 0 & 1 & 2 & 3 & 4
\end{tabular}

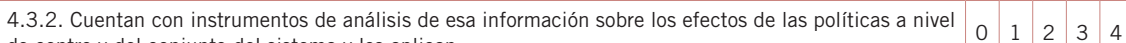
de centro y del conjunto del sistema y los aplican.

4.3.3. Están comprometidos personal y políticamente con el procesamiento de la información sobre

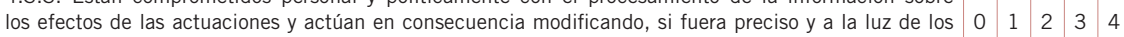
contenidos del feedback, sus decisiones previas.

\subsection{Evalúa el impacto de las políticas}

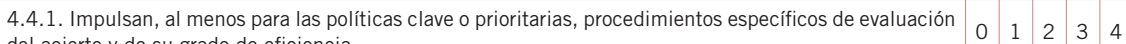
del acierto y de su grado de eficiencia.

4.4.2. Procuran la identificación de las causas de los aciertos, de los errores o de los efectos no deseados, al menos para las políticas clave.

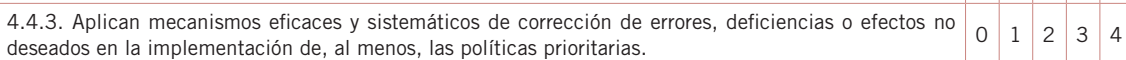

5. Gobernanza basada en la construcción de capacidades

5.1. Establece diferentes fórmulas para compartir con los actores principales el conocimiento sobre las políticas y sobre su implementación

5.1.1. Reconocen a las personas como el elemento primordial del sistema educativo.

5.1.2. Proporcionan información significativa a los actores principales del sistema, en sus distintos niveles,

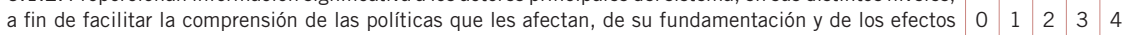
de su implementación.

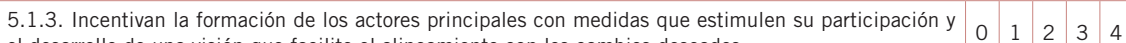
el desarrollo de una visión que facilite el alineamiento con los cambios deseados.

5.1.4. Impulsan en los centros educativos la adquisición de las habilidades para el liderazgo pedagógico y de gestión, así como para el trabajo en equipo. 
Valore el nivel de calidad de la gobernanza del sistema educativo español, a través de cada uno de los diferentes criterios que se formulan a continuación, utilizando para ello la siguiente escala: [(0) nulo, (1) escaso, (2) mediano, (3) alto, (4) muy alto].

5.2. Ayuda a los actores principales a adquirir y a utilizar información relevante, a su nivel, para el éxito en la implementación de las políticas

\begin{tabular}{|c|c|c|c|c|c|}
\hline 5.2.1. Actúan a este respecto sobre el nivel individual (padres, profesores y directores escolares). & 0 & 1 & 2 & 3 & 4 \\
\hline $\begin{array}{l}\text { 5.2.2. Operan sobre el nivel institucional apoyando particularmente a los centros educativos en formación, } \\
\text { generación de estructuras y buena gestión. }\end{array}$ & 0 & 1 & 2 & 3 & 4 \\
\hline $\begin{array}{l}\text { 5.2.3. Implementan fórmulas efectivas de apoyo a aquellos actores que actúan a nivel del conjunto del } \\
\text { sistema (responsables de las políticas, organizaciones sindicales y profesionales, confederaciones de } \\
\text { padres, sectores empresariales, productivos y de servicios, etc.). }\end{array}$ & 0 & 1 & 2 & 3 & 4 \\
\hline \multicolumn{6}{|l|}{ 6. Gobernanza vinculada a la rendición de cuentas } \\
\hline \multicolumn{6}{|l|}{ 6.1. Concede una gran importancia a la transparencia } \\
\hline $\begin{array}{l}\text { 6.1.1. Facilitan que el flujo de información relevante sobre sus actuaciones llegue a los distintos sectores } \\
\text { de la comunidad educativa y al conjunto de la sociedad. }\end{array}$ & 0 & 1 & 2 & 3 & 4 \\
\hline $\begin{array}{l}\text { 6.1.2. Dictan instrucciones para hacer de la transparencia una característica del sistema educativo, de } \\
\text { sus objetivos y de sus procedimientos, en los diferentes niveles institucionales. }\end{array}$ & 0 & 1 & 2 & 3 & 4 \\
\hline $\begin{array}{l}\text { 6.1.3. Procuran que en los niveles inferiores del sistema se desarrolle una cultura de la transparencia, sin } \\
\text { perjuicio del respeto debido a la dignidad de la persona y de la necesaria protección de datos. }\end{array}$ & 0 & 1 & 2 & 3 & 4 \\
\hline $\begin{array}{l}\text { 6.1.4. Establecen mecanismos que garantizan la transparencia, particularmente, en los procesos relacio- } \\
\text { nados con la selección de personal y la profesionalización. }\end{array}$ & 0 & 1 & 2 & 3 & 4 \\
\hline \multicolumn{6}{|l|}{ 6.2. Establece mecanismos de responsabilidad } \\
\hline $\begin{array}{l}\text { 6.2.1. Promueven o aprovechan los sistemas de evaluación de resultados para trasladar a los actores el } \\
\text { reconocimiento de los aciertos, de los posibles fallos y el compromiso con la mejora. }\end{array}$ & 0 & 1 & 2 & 3 & 4 \\
\hline $\begin{array}{l}\text { 6.2.2. Orientan sistemáticamente sus actuaciones de modo que se reduzca, en lo posible, el riesgo de } \\
\text { error sin comprometer por ello los beneficios de la innovación. }\end{array}$ & 0 & 1 & 2 & 3 & 4 \\
\hline $\begin{array}{l}\text { 6.2.3. Estimulan los mecanismos de responsabilidad tanto verticales (de acuerdo con el orden jerárquico) } \\
\text { como horizontales (entre actores del sistema sin una relación de dependencia jerárquica, propiamente } \\
\text { dicha, entre ellos). }\end{array}$ & 0 & 1 & 2 & 3 & 4 \\
\hline $\begin{array}{l}\text { 6.2.4. Establecen fórmulas de reconocimiento (administrativo, académico y/o económico) del mérito de } \\
\text { aquellos individuos e instituciones que, mediante el correcto ejercicio de sus responsabilidades, contribuyen } \\
\text { a la mejora de los procedimientos y de los resultados. }\end{array}$ & 0 & 1 & 2 & 3 & 4 \\
\hline $\begin{array}{l}\text { 6.2.5. Definen y aplican con claridad un marco para el desarrollo de la autonomía de los centros educativos } \\
\text { que comprende los procesos de rendición de cuentas. }\end{array}$ & 0 & 1 & 2 & 3 & 4 \\
\hline
\end{tabular}

